\title{
Infinite primitive directed graphs
}

\author{
Simon M. Smith
}

Received: 26 January 2006 / Accepted: 8 June 2009 / Published online: 28 July 2009

(C) Springer Science+Business Media, LLC 2009

\begin{abstract}
A group $G$ of permutations of a set $\Omega$ is primitive if it acts transitively on $\Omega$, and the only $G$-invariant equivalence relations on $\Omega$ are the trivial and universal relations.

A digraph $\Gamma$ is primitive if its automorphism group acts primitively on its vertex set, and is infinite if its vertex set is infinite. It has connectivity one if it is connected and there exists a vertex $\alpha$ of $\Gamma$, such that the induced digraph $\Gamma \backslash\{\alpha\}$ is not connected. If $\Gamma$ has connectivity one, a lobe of $\Gamma$ is a connected subgraph that is maximal subject to the condition that it does not have connectivity one. Primitive graphs (and thus digraphs) with connectivity one are necessarily infinite.

The primitive graphs with connectivity one have been fully classified by Jung and Watkins: the lobes of such graphs are primitive, pairwise-isomorphic and have at least three vertices. When one considers the general case of a primitive digraph with connectivity one, however, this result no longer holds. In this paper we investigate the structure of these digraphs, and obtain a complete characterisation.
\end{abstract}

Keywords Primitive · Graph · Digraph · Permutation · Group · Orbital graph · Orbital digraph $\cdot$ Block-cut-vertex tree

\section{Preliminaries}

Throughout this note, a graph $\Gamma$ will be a pair $(V \Gamma, E \Gamma)$, where $V \Gamma$ is the set of vertices of $\Gamma$, and $E \Gamma$ the set of edges. The set $E \Gamma$ consists of unordered pairs of distinct elements of $V \Gamma$.

A digraph $\Gamma$ is a pair $(V \Gamma, A \Gamma)$, where $A \Gamma$ is the set of arcs of $\Gamma$. Each arc is an ordered pair of distinct elements of $V \Gamma$. All paths in a digraph will be undi-

S.M. Smith $(\bowtie)$

Mathematical Institute, University of Oxford, Oxford, UK

e-mail: simon.smith@chch.oxon.org 
rected, unless otherwise stated. A directed cycle in $\Gamma$ is a path $\alpha_{0} \alpha_{1} \ldots \alpha_{n}$ such that $\left(\alpha_{n}, \alpha_{0}\right) \in A \Gamma$ and $\left(\alpha_{i}, \alpha_{i+1}\right) \in A \Gamma$ for all integers $i$ satisfying $0 \leq i<n$.

All graphs and digraphs will be free of loops and multiple edges. They are said to be infinite if their vertex sets are infinite.

The distance between two connected vertices $\alpha$ and $\beta$ in a graph or digraph $\Gamma$ will be denoted by $d_{\Gamma}(\alpha, \beta)$.

Groups, and in particular groups of automorphisms, will play a leading role in many of the arguments presented herein. Throughout this work, $G$ will be a group of permutations of a set $\Omega$, where $\Omega$ will usually be the vertex set of some infinite digraph.

If $\alpha \in \Omega$ and $g \in G$, we denote the image of $\alpha$ under $g$ by $\alpha^{g}$. Following this notation, all permutations will act on the right. The orbit of $\alpha$ under the action of $G$ will be denoted by $\alpha^{G}$.

If $\alpha \in \Omega$, we denote the stabiliser of $\alpha$ in $G$ by $G_{\alpha}$, and if $\Sigma \subseteq \Omega$ we denote the setwise and pointwise stabilisers of $\Sigma$ in $G$ by $G_{\{\Sigma\}}$ and $G_{(\Sigma)}$ respectively.

A transitive group $G$ is primitive on $\Omega$ if the only $G$-congruences admitted by $\Omega$ are the trivial and universal equivalence relations; otherwise $G$ is said to be imprimitive. It is said to act regularly on $\Omega$ if $G_{\alpha}=1$ for each $\alpha \in \Omega$.

A subset $\Delta$ of $\Omega$ is called a block if for all $g \in G$ we have either $\Delta^{g}=\Delta$ or $\Delta^{g} \cap \Delta=\emptyset$. A block is called trivial if $|\Delta|=1$, and proper if $\Delta \neq \Omega$. Since the existence of a non-trivial proper block permits the construction of a non-trivial and non-universal $G$-congruence on $\Omega$, the group $G$ is primitive if and only if $\Omega$ does not contain a non-trivial proper block.

The following is well known, and is often a very useful test for primitivity.

Theorem 1.1 [1, Theorem 4.7] If $G$ is a transitive group of permutations on $\Omega$, and $|\Omega|>1$, then $G$ is primitive on $\Omega$ if and only if, for every $\alpha \in \Omega$, the stabiliser $G_{\alpha}$ is a maximal subgroup of $G$.

A graph or digraph $\Gamma$ is primitive if its automorphism group Aut $\Gamma$ acts primitively on the set $V \Gamma$, and is automorphism-regular if Aut $\Gamma$ acts regularly on $V \Gamma$.

A primitive graph or digraph $\Gamma$ with at least one edge or arc is always connected. Indeed, the connected components of $\Gamma$ form a set of Aut $\Gamma$-congruence classes.

The connectivity of an infinite connected graph or digraph $\Gamma$ is the smallest possible size of a subset $W$ of $V \Gamma$ for which the induced graph $\Gamma \backslash W$ is disconnected. A lobe of $\Gamma$ is a connected subgraph that is maximal subject to the condition it has connectivity strictly greater than one. If $\Gamma$ has connectivity one, then the vertices $\alpha$ for which $\Gamma \backslash\{\alpha\}$ is disconnected are called the cut vertices of $\Gamma$.

\section{Local structure}

Consider the following construction. Let $V_{1}$ be the set of cut vertices of a connected graph $\Gamma$, and let $V_{2}$ be a set in bijective correspondence with the set of lobes of $\Gamma$. We let $T$ be a bipartite graph whose parts are $V_{1}$ and $V_{2}$. Two vertices $\alpha \in V_{1}$ and $x \in V_{2}$ are adjacent in $T$ if and only if $\alpha$ is contained in the lobe of $\Gamma$ corresponding 
to $x$. In fact, this construction yields a tree, which is called the block-cut-vertex tree of $\Gamma$. Note that if $\Gamma$ has connectivity one and block-cut-vertex tree $T$, then any group $G$ acting on $\Gamma$ has a natural action on $T$.

It is perhaps helpful to the reader at this point to describe a graph that is typical of those in which we are interested. Let $P_{5}$ denote the Petersen Graph. To each vertex $\alpha$ in $P_{5}$ we adjoin another two copies of $P_{5}$ in such a way that $\alpha$ is contained in three distinct copies of $P_{5}$ that intersect only in $\alpha$. We continue this process for each additional vertex whenever a new $P_{5}$ is adjoined. In this way we obtain an infinite graph with connectivity one, whose lobes are isomorphic to $P_{5}$. The block-cut-vertex tree of this graph is a biregular tree, in which one set of the natural bipartition has valency 3 , and the other valency 10 . As we shall see, this graph is primitive.

Let $\Gamma$ be a primitive digraph with connectivity one whose lobes have at least three vertices, and suppose $G$ is a vertex- and arc-transitive group of automorphisms of $\Gamma$. Since $\Gamma$ is vertex-transitive with connectivity one, every vertex is a cut vertex. Fix some lobe $\Lambda$ of $\Gamma$, and let $H$ be the subgroup of the automorphism group Aut $\Lambda$ induced by the setwise stabiliser $G_{\{\Lambda\}}$ of $V \Lambda$ in $G$. Let $T$ be the block-cut-vertex tree of $\Gamma$, and let $x$ be the vertex of $T$ that corresponds to the lobe $\Lambda$. Our aim in this section is to show $H$ is primitive but not regular.

If $x_{1}$ and $x_{2}$ are distinct vertices of the tree $T$, we use $C\left(T \backslash\left\{x_{1}\right\}, x_{2}\right)$ to denote the connected component of $T \backslash\left\{x_{1}\right\}$ that contains the vertex $x_{2}$.

Lemma 2.1 If $G$ acts primitively on the vertices of $\Gamma$, then $H$ acts primitively on the vertices of $\Lambda$.

Proof If $H$ acts transitively but not primitively on $V \Lambda$, then there exists a nontrivial proper block $\Delta \subseteq V \Lambda$. For any two distinct vertices $\alpha, \beta \in \Delta$, the digraph $\left(V \Lambda,(\alpha, \beta)^{H}\right)$ is not connected, since it does not contain a path from $\alpha$ to any vertex in $V \Lambda \backslash \Delta$.

If $H$ does not act transitively on $V \Lambda$, then one may choose distinct vertices $\alpha, \beta, \gamma \in V \Lambda$ such that $\beta \in \alpha^{H}$ but $\gamma \notin \alpha^{H}$. Again the digraph $\left(V \Lambda,(\alpha, \beta)^{H}\right)$ is not connected, as there is no path from $\alpha$ to $\gamma$. Thus, if $H$ is not transitive on $V \Lambda$, or if $H$ is transitive but not primitive on $V \Lambda$, then there exist distinct vertices $\alpha, \beta \in V \Lambda$ such that the digraph $\Lambda^{\prime}:=\left(V \Lambda,(\alpha, \beta)^{H}\right)$ is not connected.

Suppose this is the case, and choose distinct vertices $\alpha, \beta \in V \Lambda$ such that $\Lambda^{\prime}$ is not connected. We will show this assumption implies the digraph $\Gamma^{\prime}:=\left(V \Gamma,(\alpha, \beta)^{G}\right)$ cannot be connected, and is therefore not primitive; whence $G$ cannot be primitive.

Recall that $T$ is the block-cut-vertex tree of $\Gamma$ and $x$ is the vertex of $T$ corresponding to the lobe $\Lambda$. Let $\left\{\Delta_{i}\right\}_{i \in I}$ be the set of connected components of $\Lambda^{\prime}$ and let

$$
\mathcal{C}_{i}:=\bigcup_{\delta \in \Delta_{i}} C(T \backslash\{x\}, \delta) \cap V \Gamma .
$$

Suppose $\delta_{i} \in \mathcal{C}_{i}$ and $\delta_{j} \in \mathcal{C}_{j}$, with $i \neq j$. We claim $\delta_{i}$ and $\delta_{j}$ are not adjacent in $\Gamma^{\prime}$. Indeed, since the distance $d_{T}(\alpha, \beta)$ between $\alpha$ and $\beta$ in $T$ is equal to 2 , if $\delta_{i}$ and $\delta_{j}$ are to be adjacent in the arc-transitive digraph $\Gamma^{\prime}$, it must be the case that $d_{T}\left(\delta_{i}, \delta_{j}\right)=2$. If either $\delta_{i}$ or $\delta_{j}$ is not adjacent to $x$ in $T$ then $d_{T}\left(\delta_{i}, \delta_{j}\right)>2$, so they 
cannot be adjacent in $\Gamma^{\prime}$. On the other hand, if $\delta_{i}$ and $\delta_{j}$ are adjacent to $x$ in $T$, then they both lie in $V \Lambda=V \Lambda^{\prime}$, and therefore $\delta_{i} \in \Delta_{i}$ and $\delta_{j} \in \Delta_{j}$. In this case, if they are adjacent in $\Gamma^{\prime}$ then there exists $g \in G$ such that either $\left(\delta_{i}, \delta_{j}\right)$ or $\left(\delta_{j}, \delta_{i}\right)$ is equal to $(\alpha, \beta)^{g}$. Such an automorphism must fix $V \Lambda$ setwise, and therefore lies in $G_{\{\Lambda\}}$. Thus, there exists an element $h \in H$ such that either $\left(\delta_{i}, \delta_{j}\right)$ or $\left(\delta_{j}, \delta_{i}\right)$ is equal to $(\alpha, \beta)^{h}$, meaning that $\delta_{i}$ and $\delta_{j}$ are adjacent in $\Lambda^{\prime}$; however, this contradicts the fact that $\delta_{i}$ and $\delta_{j}$ are in distinct components of $\Lambda^{\prime}$. Hence, $\delta_{i}$ and $\delta_{j}$ are not adjacent in $\Gamma^{\prime}$.

Hence, there can be no path in $\Gamma^{\prime}$ between a vertex in $\mathcal{C}_{i}$ and a vertex in $\mathcal{C}_{j}$ whenever $i \neq j$, and so the digraph $\Gamma^{\prime}$ is not connected. Whence, $\Gamma^{\prime}$ cannot be primitive, and $G$ cannot act primitively on $V \Gamma$.

Fix distinct vertices $\alpha, \beta \in V \Gamma$ and recall that $\alpha$ and $\beta$ are also vertices of the block-cut-vertex tree $T$.

A geodesic between two vertices is a shortest path between them. In a tree, there is a unique geodesic between any two vertices. Let $[\alpha, \beta]_{T}$ be the $T$-geodesic between $\alpha$ and $\beta$, and let $(\alpha, \beta)_{T}$ be the $T$-geodesic $[\alpha, \beta]_{T}$ excluding both $\alpha$ and $\beta$. This notation extends obviously to $[\alpha, \beta)_{T}$ and $(\alpha, \beta]_{T}$.

Since $\alpha$ and $\beta$ are vertices of both $\Gamma$ and $T$, the distance $d_{T}(\alpha, \beta)$ is even, so we may choose a vertex $y \in(\alpha, \beta)_{T}$ that is distinct from $\alpha$ and $\beta$.

Lemma 2.2 If $g \in G_{\alpha}$ does not fix $y \in V T$, and $\delta \notin C(T \backslash\{y\}, \alpha)$, then $\delta^{g} \notin C(T \backslash$ $\{y\}, \beta)$.

Proof If $\delta \notin C(T \backslash\{y\}, \alpha)$ and $\delta^{g} \in C(T \backslash\{y\}, \beta)$ then $\delta, \delta^{g} \notin C(T \backslash\{y\}, \alpha)$, so we must have $g \in G_{\alpha, y}$.

Lemma 2.3 If $g \in G_{\alpha}$ does not fix the vertex $y$ and $\delta \notin C(T \backslash\{y\}, \alpha)$ then $d_{T}\left(y, \delta^{g}\right)>d_{T}(y, \delta)$.

Proof If $\delta \notin C(T \backslash\{y\}, \alpha)$ then $y \in[\alpha, \delta]_{T}$. Thus $d_{T}\left(\delta, \delta^{g}\right)=d_{T}(\delta, y)+d_{T}\left(y, y^{g}\right)+$ $d_{T}\left(y^{g}, \delta^{g}\right)$ and $d_{T}\left(\delta, \delta^{g}\right)=d_{T}(\delta, y)+d_{T}\left(y, \delta^{g}\right)$. Therefore $d_{T}\left(y, \delta^{g}\right)=d_{T}\left(y^{g}, \delta^{g}\right)+$ $d_{T}\left(y, y^{g}\right)$. Now $d_{T}\left(y^{g}, \delta^{g}\right)=d_{T}(y, \delta)$, and $d_{T}\left(y, y^{g}\right) \geq 1$. Whence $d_{T}\left(y, \delta^{g}\right)>$ $d_{T}(y, \delta)$.

Henceforth, if $H$ is a subgroup of $G$, then we will write $H \leq G$; if we wish to exclude the possibility of $H=G$ we will instead write $H<G$.

Lemma 2.4 Let $g_{1}, \ldots, g_{n} \in G_{\alpha}$ and $h_{1}, \ldots, h_{n} \in G_{\beta}$, and suppose $G_{\alpha, y}=G_{\beta, y}$. If there exists $\gamma \in V T$ such that $G_{\alpha, y} \leq G_{\gamma}$ then, for some $m \leq n$, there exist $g_{2}^{\prime}, \ldots, g_{m}^{\prime} \in G_{\alpha} \backslash G_{y}$ and $g_{1}^{\prime} \in G_{\alpha} \backslash G_{y} \cup\{1\}$ together with $h_{1}^{\prime}, \ldots, h_{m-1}^{\prime} \in G_{\beta} \backslash G_{y}$ and $h_{m}^{\prime} \in G_{\beta} \backslash G_{y} \cup\{1\}$ such that

$$
\gamma^{g_{1}^{\prime} h_{1}^{\prime} \ldots g_{m}^{\prime} h_{m}^{\prime}}=\gamma^{g_{1} h_{1} \ldots g_{n} h_{n}}
$$

Proof The proof of this lemma will be an inductive argument. Suppose there exists $\gamma \in V T$ such that $G_{\alpha, y} \leq G_{\gamma}$. 
Let $n=1$. When considering $h_{1} \in G_{\beta}$ we have two cases: either $h_{1} \in G_{y}$ or $h_{1} \in$ $G_{\beta} \backslash G_{y}$. If $h_{1} \in G_{y}$ then $h_{1} \in G_{\beta, y}=G_{\alpha, y}$, so $g_{1} h_{1} \in G_{\alpha}$. In this case, redefine $g_{1}:=g_{1} h_{1}$ and set $h_{1}^{\prime}:=1$. Alternatively, if $h_{1} \in G_{\beta} \backslash G_{y}$ then set $h_{1}^{\prime}:=h_{1}$. Having found a suitable $h_{1}^{\prime}$, we will now construct $g_{1}^{\prime}$ from the (possibly redefined) element $g_{1} \in G_{\alpha}$. We again have two cases: either $g_{1} \in G_{y}$ or $g_{1} \in G_{\alpha} \backslash G_{y}$. If $g_{1} \in G_{y}$ then $g_{1} \in G_{\alpha, y}$ and so $g_{1} \in G_{\gamma}$. In this case we can choose $g_{1}^{\prime}:=1$. Otherwise, if $g_{1} \in G_{\alpha} \backslash G_{y}$, then choose $g_{1}^{\prime}:=g_{1}$. In choosing $g_{1}^{\prime}$ and $h_{1}^{\prime}$ in this way we ensure that

$$
\gamma^{g_{1} h_{1}}=\gamma^{g_{1}^{\prime} h_{1}^{\prime}}
$$

so the hypothesis holds when $n=1$.

Let $k$ be a positive integer, and suppose the hypothesis is true for all integers $n \leq k$. Fix $g_{1}, \ldots, g_{k+1} \in G_{\alpha}$ and $h_{1}, \ldots, h_{k+1} \in G_{\beta}$, and set

$$
\gamma^{\prime}:=\gamma^{g_{1} h_{1} \ldots g_{k+1} h_{k+1}} .
$$

We will use induction to construct elements $g_{2}^{\prime}, \ldots, g_{m}^{\prime} \in G_{\alpha} \backslash G_{y}$ and $g_{1}^{\prime} \in G_{\alpha} \backslash$ $G_{y} \cup\{1\}$ together with $h_{1}^{\prime}, \ldots, h_{m-1}^{\prime} \in G_{\beta} \backslash G_{y}$ and $h_{m}^{\prime} \in G_{\beta} \backslash G_{y} \cup\{1\}$ such that

$$
\gamma^{g_{1}^{\prime} h_{1}^{\prime} \ldots g_{m}^{\prime} h_{m}^{\prime}}=\gamma^{\prime},
$$

where $m$ is some integer less than or equal to $k+1$.

We begin by considering $h_{k+1} \in G_{\beta}$. There are two cases: either $h_{k+1} \in G_{y}$ or $h_{k+1} \in G_{\beta} \backslash G_{y}$. If $h_{k+1} \in G_{y}$ then $h_{k+1} \in G_{\beta, y}=G_{\alpha, y}$, so $g_{k+1} h_{k+1} \in G_{\alpha}$. In this case, redefine $g_{k+1}:=g_{k+1} h_{k+1}$ and set $h^{\prime}:=1$. If, on the other hand, $h_{k+1} \in$ $G_{\beta} \backslash G_{y}$, then set $h^{\prime}:=h_{k+1}$.

If we now consider the (possibly redefined) element $g_{k+1} \in G_{\alpha}$, there are again two cases: either $g_{k+1} \in G_{y}$, or $g_{k+1} \in G_{\alpha} \backslash G_{y}$. If $g_{k+1} \in G_{y}$ then $g_{k+1} \in G_{\alpha, y}=$ $G_{\beta, y}$, so $h_{k} g_{k+1} h^{\prime} \in G_{\beta}$. In this case, let $h^{\prime \prime}:=h_{k} g_{k+1} h^{\prime}$; then

$$
\gamma^{\prime}=\gamma^{g_{1} h_{1} \ldots g_{k} h^{\prime \prime}}
$$

so we can apply the induction hypothesis to $\gamma^{g_{1} h_{1} \ldots g_{k} h^{\prime \prime}}$ and we are done. If, on the other hand, $g_{k+1} \in G_{\alpha} \backslash G_{y}$, then set $g^{\prime}:=g_{k+1}$, and observe

$$
\gamma^{\prime}=\gamma^{g_{1} h_{1} \ldots g_{k} h_{k} g^{\prime} h^{\prime}}
$$

By the induction hypothesis, for some $l \leq k$ there exist $g_{2}^{\prime}, \ldots, g_{l}^{\prime} \in G_{\alpha} \backslash G_{y}$ and $g_{1}^{\prime} \in G_{\alpha} \backslash G_{y} \cup\{1\}$ together with $h_{1}^{\prime}, \ldots, h_{l-1}^{\prime} \in G_{\beta} \backslash G_{y}$ and $h_{l}^{\prime} \in G_{\beta} \backslash G_{y} \cup\{1\}$ such that

$$
\gamma^{g_{1} h_{1} \ldots g_{k} h_{k}}=\gamma^{g_{1}^{\prime} h_{1}^{\prime} \ldots g_{l}^{\prime} h_{l}^{\prime}}
$$

At this final stage in the proof, we again face two possibilities: either $h_{l}^{\prime}=1$ or $h_{l}^{\prime} \in G_{\beta} \backslash G_{y}$. In the first instance define $g^{\prime \prime}:=g_{l}^{\prime} h_{l}^{\prime} g^{\prime}$, so $\gamma^{\prime}=\gamma^{g_{1}^{\prime} h_{1}^{\prime} \ldots g_{l-1}^{\prime} h_{l-1}^{\prime} g^{\prime \prime} h^{\prime}}$. Since $g^{\prime \prime}=g_{l}^{\prime} g^{\prime} \in G_{\alpha}$ and $h^{\prime} \in G_{\beta}$ and $l \leq k$, we may apply the induction hypothesis.

On the other hand, if $h_{l}^{\prime} \in G_{\beta} \backslash G_{y}$, then set $g_{l+1}^{\prime}:=g^{\prime} \in G_{\alpha} \backslash G_{y}$ and $h_{l+1}^{\prime}:=$ $h^{\prime} \in G_{\beta} \backslash G_{y} \cup\{1\}$, and observe $\gamma^{\prime}=\gamma^{g_{1}^{\prime} h_{1}^{\prime} \ldots g_{l+1}^{\prime} h_{l+1}^{\prime}}$. Now $l \leq k$, so defining $m$ to be 
$l+1$ we have $m \leq k+1$. Thus in both cases the hypothesis holds. It is therefore true for $n=k+1$.

We are now in a position to present the main result of this section which describes necessary conditions for a vertex-transitive subgroup of the automorphism group of an infinite primitive digraph with connectivity one to be imprimitive.

Theorem 2.5 Let $G$ be a vertex-transitive group of automorphisms of a connectivityone digraph $\Gamma$ whose lobes have at least three vertices, and let $T$ be the block-cutvertex tree of $\Gamma$. If there exist distinct vertices $\alpha, \beta \in V \Gamma$ such that, for some vertex $x \in(\alpha, \beta)_{T}$,

$$
G_{\alpha, x}=G_{\beta, x},
$$

then $G$ does not act primitively on $V \Gamma$.

Proof Suppose $G$ acts primitively on $V \Gamma$ and there exist distinct vertices $\alpha, \beta \in V \Gamma$ and $x \in(\alpha, \beta)_{T}$ such that $G_{\alpha, x}=G_{\beta, x}$. We will begin by showing the group $\left\langle G_{\alpha}, G_{\beta}\right\rangle$ generated by $G_{\alpha}$ and $G_{\beta}$ is not equal to $G$; then we shall show it is not equal to $G_{\alpha}$. Whence, $G_{\alpha}<\left\langle G_{\alpha}, G_{\beta}\right\rangle<G$ which, by applying Theorem 1.1, will contradict the assumption that $G$ is primitive.

Without loss of generality, suppose $d_{T}(x, \alpha) \leq d_{T}(x, \beta)$. If the orbit $\beta^{\left\langle G_{\alpha}, G_{\beta}\right\rangle}$ contains $\alpha$, then there exist elements $g_{1}, \ldots, g_{n} \in G_{\alpha}$ and $h_{1}, \ldots, h_{n} \in G_{\beta}$ such that $\alpha=\beta^{g_{1} h_{1} \ldots g_{n} h_{n}}$. By Lemma 2.4, we can find $m \leq n$ and $g_{2}^{\prime}, \ldots, g_{m}^{\prime} \in G_{\alpha} \backslash G_{x}$ and $g_{1}^{\prime} \in G_{\alpha} \backslash G_{x} \cup\{1\}$ together with $h_{1}^{\prime}, \ldots, h_{m-1}^{\prime} \in G_{\beta} \backslash G_{x}$ and $h_{m}^{\prime} \in G_{\beta} \backslash G_{x} \cup\{1\}$ such that

$$
\alpha=\beta^{g_{1}^{\prime} h_{1}^{\prime} \ldots g_{m}^{\prime} h_{m}^{\prime}}
$$

Suppose these automorphisms are chosen so that $m$ is minimal.

Now either $g_{1}^{\prime} \in G_{\alpha} \backslash G_{x}$ or $g_{1}^{\prime}=1$. If $g_{1}^{\prime}=1$ then $\beta^{g_{1}^{\prime}}=\beta$ and therefore $\beta^{g_{1}^{\prime} h_{1}^{\prime}}=$ $\beta$. Thus $\beta^{g_{2}^{\prime} h_{2}^{\prime} \ldots g_{m}^{\prime} h_{m}^{\prime}}=\alpha$, contradicting the minimality of $m$. So we must have $g_{1}^{\prime} \in$ $G_{\alpha} \backslash G_{x}$. Since $\beta \notin C(T \backslash\{x\}, \alpha)$, we may apply Lemma 2.2 and Lemma 2.3 to obtain $d_{T}\left(x, \beta^{g_{1}^{\prime}}\right)>d_{T}(x, \beta)$ and $\beta^{g_{1}^{\prime}} \notin C(T \backslash\{x\}, \beta)$.

We now observe $h_{1}^{\prime} \neq 1$. Indeed, if $h_{1}^{\prime}=1$ then $m=1$ and $\alpha=\beta^{g_{1}^{\prime}}$; since $g_{1}^{\prime} \in G_{\alpha}$ this is clearly not possible.

Thus, $h_{1}^{\prime} \in G_{\beta} \backslash G_{x}$ and $\beta^{g_{1}^{\prime}} \notin C(T \backslash\{x\}, \beta)$, and we can again deduce from Lemma 2.2 and Lemma 2.3 that $d_{T}\left(x, \beta^{g_{1}^{\prime} h_{1}^{\prime}}\right)>d_{T}\left(x, \beta^{g_{1}^{\prime}}\right)>d_{T}(x, \beta)$, and $\beta^{g_{1}^{\prime} h_{1}^{\prime}} \notin$ $C(T \backslash\{x\}, \alpha)$.

We may continue to apply Lemmas 2.2 and 2.3 to obtain $\beta^{g_{1}^{\prime} h_{1}^{\prime} \ldots g_{m}^{\prime}} \notin C(T \backslash\{x\}, \beta)$ and $d_{T}\left(x, \beta^{g_{1}^{\prime}} h_{1}^{\prime} \ldots g_{m}^{\prime}\right)>d_{T}(x, \beta)$. Now either $h_{m}^{\prime} \in G_{\beta} \backslash G_{x}$ or $h_{m}^{\prime}=1$. If $h_{m}^{\prime}=1$ then $\alpha=\beta^{g_{1}^{\prime} h_{1}^{\prime} \ldots g_{m}^{\prime}}$, and so $d_{T}(x, \alpha)=d_{T}\left(x, \beta^{g_{1}^{\prime} h_{1}^{\prime} \ldots g_{m}^{\prime}}\right)>d_{T}(x, \beta)$. If $h_{m}^{\prime} \in G_{\beta} \backslash G_{x}$ then, by Lemma $2.3, d_{T}\left(x, \beta^{g_{1}^{\prime}} h_{1}^{\prime} \ldots g_{m}^{\prime} h_{m}^{\prime}\right)>d_{T}(x, \beta)$; that is, $d_{T}(x, \alpha)>d_{T}(x, \beta)$. Thus, in both cases $d_{T}(x, \alpha)>d_{T}(x, \beta)$. This contradicts our assumption that $d_{T}(x, \alpha) \leq$ $d_{T}(x, \beta)$. Hence $\alpha \notin \beta^{\left\langle G_{\alpha}, G_{\beta}\right\rangle}$, and so $\left\langle G_{\alpha}, G_{\beta}\right\rangle$ cannot act transitively on the set $V \Gamma$. This ensures that $\left\langle G_{\alpha}, G_{\beta}\right\rangle \neq G$.

By Theorem 1.1, we must therefore have $\left\langle G_{\alpha}, G_{\beta}\right\rangle=G_{\alpha}$. Thus, the set of vertices $\operatorname{Fix}\left(G_{\alpha}\right)$ fixed by $G_{\alpha}$ contains both $\alpha$ and $\beta$. This set is a block of imprimitivity. Thus, 
every vertex in $V \Gamma$ must be fixed by $G_{\alpha}$, and so $G_{\alpha}=\langle 1\rangle$. However, $G_{\alpha}$ is a maximal subgroup of $G$, so $G_{\alpha}=\langle 1\rangle$ implies that $G$ is a finite cyclic group of prime order. This, however, is impossible, as $G$ acts transitively on the infinite set $V \Gamma$.

Hence $\left\langle G_{\alpha}, G_{\beta}\right\rangle \neq G_{\alpha}$, and $\left\langle G_{\alpha}, G_{\beta}\right\rangle \neq G$, which contradicts our assumption that $G$ is primitive.

Theorem 2.6 Let $G$ be a vertex-transitive group of automorphisms of a connectivityone digraph $\Gamma$ whose lobes have at least three vertices. If $G$ acts primitively on $V \Gamma$ and $\Lambda$ is some lobe of $\Gamma$ then $G_{\{\Lambda\}}$ is primitive and not regular on $V \Lambda$.

Proof Suppose $G$ acts primitively on $V \Gamma$, and $\Lambda$ is a lobe of $\Gamma$. By Lemma 2.1, $G_{\{\Lambda\}}$ acts primitively on $V \Lambda$. Suppose this action is regular. If $T$ is the block-cutvertex tree of $\Gamma$ then there exists a vertex $x \in V T$ corresponding to the lobe $\Lambda$. Choose distinct vertices $\alpha$ and $\beta$ in $V \Lambda$, and observe $G_{\alpha, x}=G_{\alpha,\{\Lambda\}} \leq G_{\beta}$ and $G_{\beta, x}=G_{\beta,\{\Lambda\}} \leq G_{\alpha}$; furthermore, $x \in(\alpha, \beta)_{T}$. This, however, is impossible, as it implies $G$ is imprimitive by Theorem 2.5.

\section{Global structure}

In this section we shall employ Theorem 2.6 to give a complete characterisation of the primitive connectivity-one digraphs.

Lemma 3.1 Suppose $\Gamma$ is a vertex-transitive digraph with connectivity one, whose lobes are vertex-transitive, have at least three vertices and are pairwise isomorphic.

If $\gamma$ is a vertex in some lobe $\Lambda$ of $\Gamma$ and $\alpha \in V \Gamma$ is $\gamma$ or lies in a component of $\Gamma \backslash\{\gamma\}$ distinct from the component containing $V \Lambda \backslash\{\gamma\}$, then the subgroup of Aut $\Lambda$ induced by the action of (Aut $\Gamma)_{\alpha,\{\Lambda\}}$ on $\Lambda$ is (Aut $\left.\Lambda\right)_{\gamma}$, and the group induced by the action of (Aut $\Gamma)_{\{\Lambda\}}$ on $\Lambda$ is Aut $\Lambda$.

Proof Let $T$ denote the block-cut-vertex tree of $\Gamma$, and let $\Lambda$ be a lobe of $\Gamma$. Choose $\gamma \in V \Lambda$ and let $C^{\prime}$ be a component of $\Gamma \backslash\{\gamma\}$ distinct from that which contains $V \Lambda \backslash\{\gamma\}$. Let $C$ be the subgraph of $\Gamma$ induced by $C^{\prime} \cup\{\gamma\}$, and suppose $\alpha$ is any vertex in $C$.

We begin by asserting that if $\Lambda_{1}$ and $\Lambda_{2}$ are lobes of $\Gamma$, and $\alpha_{1}$ and $\alpha_{2}$ are vertices in $\Lambda_{1}$ and $\Lambda_{2}$ respectively, then there exists an isomorphism $\rho: \Lambda_{1} \rightarrow \Lambda_{2}$ such that $\alpha_{1}^{\rho}=\alpha_{2}$. Indeed, by assumption there exists an isomorphism $\rho^{\prime}: \Lambda_{1} \rightarrow \Lambda_{2}$. Define $\alpha_{1}^{\prime}:=\alpha_{1}^{\rho^{\prime}}$. Since the lobe $\Lambda_{2}$ is vertex-transitive, there exists an automorphism $\tau$ of $\Lambda_{2}$ such that $\alpha_{1}^{\prime \tau}=\alpha_{2}$. Let $\rho:=\rho^{\prime} \tau$. Then $\rho: \Lambda_{1} \rightarrow \Lambda_{2}$ is an isomorphism, with $\alpha_{1}^{\rho}=\alpha_{1}^{\rho^{\prime} \tau}=\alpha_{1}^{\prime \tau}=\alpha_{2}$.

Let $x$ be the vertex of $T$ that corresponds to $\Lambda$. For $k \geq 0$, define $\Gamma_{k}$ to be the subgraph of $\Gamma$ induced by the set $\left\{\xi \in V \Gamma \mid d_{T}(x, \xi) \leq 2 k+1\right\}$, and $C_{k}:=C \cap \Gamma_{k}$. Note that $\Gamma_{0}=\Lambda$. We will show any automorphism $\sigma_{k}: \Gamma_{k} \rightarrow \Gamma_{k}$ which fixes $C_{k}$ admits an extension $\sigma_{k+1}: \Gamma_{k+1} \rightarrow \Gamma_{k+1}$ which fixes $C_{k+1}$.

Fix $k \geq 0$ and let $\sigma_{k}: \Gamma_{k} \rightarrow \Gamma_{k}$ be an automorphism that fixes $C_{k}$ pointwise; in particular, $\sigma_{0} \in(\text { Aut } \Lambda)_{\gamma}$. Let $\left\{\alpha_{i}\right\}_{i \in I}$ be the set of vertices in $V \Gamma_{k} \backslash V \Gamma_{k-1}$ (where 
$\left.V \Gamma_{-1}:=\emptyset\right)$. Each vertex $\alpha_{i}$ belongs to a unique lobe $\Lambda_{i}$ of $\Gamma_{k}$, and, if $k \geq 1$, the lobe $\Lambda_{i}$ possesses precisely one vertex in $\Gamma_{k-1}$. Since $\Gamma$ is vertex transitive, any two vertices lie in the same number of lobes of $\Gamma$, so let $\left\{\Lambda_{i, j}\right\}_{j \in J}$ be the set of lobes of $\Gamma$ that contain $\alpha_{i}$ and are distinct from $\Lambda_{i}$. Each lobe $\Lambda_{i, j}$ is wholly contained in $\Gamma_{k+1}$ and has exactly one vertex in $\Gamma_{k}$, namely $\alpha_{i}$. If $i \in I$, set $\alpha_{i}^{\prime}:=\alpha_{i}^{\sigma_{k}}$ and $\Lambda_{i}^{\prime}:=\Lambda_{i}^{\sigma_{k}}$. Then $\Lambda_{i}^{\prime}=\Lambda_{i^{\prime}}$ for some $i^{\prime} \in I$. For all $j \in J$ there exists an isomorphism $\rho_{i, j}$ : $\Lambda_{i, j} \rightarrow \Lambda_{i^{\prime}, j}$ such that $\alpha_{i}^{\rho_{i, j}}=\alpha_{i}^{\prime}$. Thus, we may define a mapping $\sigma_{k+1}: \Gamma_{k+1} \rightarrow$ $\Gamma_{k+1}$ with

$$
\beta^{\sigma_{k+1}}:= \begin{cases}\beta^{\sigma_{k}} & \text { if } \beta \in V \Gamma_{k} ; \\ \beta & \text { if } \beta \in C ; \\ \beta^{\rho_{i, j}} & \text { if } \beta \in V \Lambda_{i, j} \backslash C .\end{cases}
$$

This is clearly a well-defined automorphism of $\Gamma_{k+1}$.

Hence if $\sigma_{0} \in(\text { Aut } \Lambda)_{\gamma}$, then we may extend it to an automorphism $\sigma$ of $\Gamma$ that fixes $C$ pointwise, and therefore fixes $\alpha$. Since each automorphism in (Aut $\Lambda)_{\gamma}$ may be extended in this way, the subgroup of Aut $\Lambda$ induced by (Aut $\Gamma)_{\alpha,\{\Lambda\}}$ must contain (Aut $\Lambda)_{\gamma}$. Clearly no automorphism of $\Gamma$ may fix $\alpha$ and $\Lambda$ setwise whilst not also fixing $\gamma$, so these two groups must in fact be equal.

We now adjust the above argument to show that the group induced by the action of $(\text { Aut } \Gamma)_{\{\Lambda\}}$ on $\Lambda$ is Aut $\Lambda$. Fix $k \geq 0$ and consider an automorphism $\sigma_{k}: \Gamma_{k} \rightarrow \Gamma_{k}$ that fixes $V \Lambda$ setwise; in particular, $\sigma_{0} \in($ Aut $\Lambda)$.

Using the above notation, we may define a map $\sigma_{k+1}: \Gamma_{k+1} \rightarrow \Gamma_{k+1}$ with

$$
\beta^{\sigma_{k+1}}:= \begin{cases}\beta^{\sigma_{k}} & \text { if } \beta \in V \Gamma_{k} \\ \beta^{\rho_{i, j}} & \text { if } \beta \in V \Lambda_{i, j} .\end{cases}
$$

This is a well-defined automorphism of $\Gamma_{k+1}$. Thus we may extend any automorphism $\sigma_{0} \in$ (Aut $\Lambda$ ) to an automorphism $\sigma$ of $\Gamma$ that fixes $\Lambda$ setwise. Whence the group induced by the action of $(\text { Aut } \Gamma)_{\{\Lambda\}}$ on $\Lambda$ is Aut $\Lambda$.

The primitive graphs with connectivity one have the following complete characterisation.

Theorem 3.2 [3, Theorem 4.2] If $\Gamma$ is a vertex-transitive graph with connectivity one, then it is primitive if and only if the lobes of $\Gamma$ are primitive, pairwise isomorphic and each has at least three vertices.

Jung and Watkins' result, while impressive, cannot be applied to primitive digraphs without some modification. Indeed, consider the following counterexample. Let $\Gamma$ be the connectivity-one primitive graph whose lobes are undirected 3-cycles, in with each vertex lies in precisely two lobes. It is of course possible to verify this graph is primitive using Theorem 3.2.

We assign to each vertex in $\Gamma$ the label 1,2 or 3 in such a way that no two vertices in a common lobe of $\Gamma$ share the same label. Whence, each lobe of $\Gamma$ has a vertex labelled 1, a vertex labelled 2 and a vertex labelled 3. 
For each lobe in $\Gamma$ we replace its edge set with a set of three arcs, from the vertex labelled $i$ to the vertex labelled $(i+1) \bmod 3$, for $i=1,2,3$. In this way we obtain a vertex-transitive connected digraph $\Gamma^{\prime}$ whose vertex set is $V \Gamma$. Furthermore, the lobes of this digraph are primitive, pairwise isomorphic, and have at least three vertices. However, the set of vertices labelled 1 is a non-trivial proper block of the group Aut $\Gamma^{\prime}$, so this group cannot act primitively on the digraph $\Gamma^{\prime}$.

The approach taken by Jung and Watkins in their proof of Theorem 3.2 is broadly similar to the argument presented thus far. They first prove that any automorphism of a lobe $\Lambda$ of a vertex-transitive graph $\Gamma$ with connectivity one may be extended to an automorphism of $\Gamma$. It is then shown that if the lobes of $\Gamma$ are vertex primitive, have at least three vertices, and are pairwise isomorphic, then $\Gamma$ is primitive. It is here that their proof fails to apply to digraphs; by citing Theorem 1 of [2] they claim the lobes of $\Gamma$ cannot be automorphism-regular. While this is indeed true of graphs, it is not true of digraphs, as our previous example illustrates.

Imrich's result states that the automorphism group of a graph with more than two vertices cannot be regular and primitive. It relies on two results, Lemmas 2 and 3 of [2]. Lemma 2 is the well-known result that a regular primitive group of permutations must be cyclic; Lemma 3 states that any transitive abelian automorphism group of a non-trivial graph is the direct product of two cyclic groups of order 2 . Any primitive and regular automorphism group of a graph must therefore equal this direct product; Imrich shows that no such graph exists, and correctly deduces that automorphismregular primitive graphs are not possible. It is in the proof of the latter lemma that Imrich's result ceases to be applicable to digraphs: his argument requires the existence of a specific graph automorphism $\psi$. On inspection it transpires that $\psi$ is not a digraph automorphism, since it reverses the direction of edges. Thus Theorem 1 of [2] is not applicable to digraphs, which in turn causes Jung and Watkins' result to fail.

Although their result does not extend immediately to digraphs, a complete characterisation is still possible.

Theorem 3.3 If $\Gamma$ is a vertex-transitive digraph with connectivity one, then it is primitive if and only if the lobes of $\Gamma$ are primitive but not automorphism-regular, pairwise isomorphic and each has at least three vertices.

Proof Let $\Gamma$ be a vertex-transitive digraph with connectivity one. Suppose the lobes of $\Gamma$ are primitive but not automorphism-regular, pairwise isomorphic and each has at least three vertices. Let $\approx$ be an Aut $\Gamma$-congruence on $V \Gamma$ such that there exist distinct vertices $\alpha, \beta \in V \Gamma$ with $\alpha \approx \beta$. We will show this relation must be universal, and thus that $\Gamma$ is a primitive digraph.

Let $T$ be the block-cut-vertex tree of $\Gamma$, let $\gamma \in V \Gamma$ be the vertex in the geodesic $[\alpha, \beta]_{T}$ such that $d_{T}(\beta, \gamma)=2$, and let $\Lambda$ be the lobe of $\Gamma$ containing $\beta$ and $\gamma$.

By Lemma 3.1 the group (Aut $\Gamma)_{\{\Lambda\}}$ acts primitively and not regularly on the lobe $\Lambda$. Thus there exits an automorphism $h \in(\text { Aut } \Gamma)_{\gamma,\{\Lambda\}}$ which does not fix $\beta$. By restricting the action of $h$ to the vertices of $\Lambda$, we see that there must be an element in Aut $\Lambda_{\gamma}$ which does not fix $\beta$. By Lemma 3.1, the subgroup of Aut $\Lambda$ induced by $(\text { Aut } \Gamma)_{\alpha,\{\Lambda\}}$ is equal to (Aut $\left.\Lambda\right)_{\gamma}$, so there must therefore exist an element $g \in(\text { Aut } \Gamma)_{\alpha, \gamma,\{\Lambda\}}$ that does not fix $\beta$. 
Thus, $\beta$ and $\beta^{g}$ are distinct vertices in $\Lambda$. Now $\alpha \approx \beta$, so $\alpha \approx \beta^{g}$, and therefore $\beta \approx \beta^{g}$. Since $(\text { Aut } \Gamma)_{\{\Lambda\}}$ is primitive on $V \Lambda$ and $\approx$ induces a non-trivial (Aut $\left.\Gamma\right)_{\{\Lambda\}}$ congruence on $V \Lambda$, this relation must be universal in $\Lambda$. By assumption, Aut $\Gamma$ acts transitively on the lobes of $\Gamma$, so if two vertices lie in the same lobe then they must lie in the same congruence class. Thus, if $\gamma$ is any vertex of $\Gamma$, and $\alpha x_{1} \alpha_{1} x_{2} \ldots x_{n} \gamma$ is the geodesic in $T$ between $\alpha$ and $\gamma$, then $\alpha$ and $\alpha_{1}$ lie in a common lobe, so $\alpha \approx \alpha_{1}$. Similarly, $\alpha_{1} \approx \alpha_{2}$ and $\alpha_{2} \approx \alpha_{3}$, so $\alpha \approx \alpha_{2}$ and $\alpha \approx \alpha_{3}$. Continuing in this way we eventually obtain $\alpha \approx \gamma$. Hence, this congruence relation is universal on $V \Gamma$.

Conversely, suppose the group Aut $\Gamma$ acts primitively on $V \Gamma$. Since $\Gamma$ is a primitive digraph with connectivity one, we can obtain an graph $\Gamma^{\prime}$ with vertex set $V \Gamma$ and edge set $\{\{\alpha, \beta\} \mid(\alpha, \beta) \in A \Gamma\}$. Two vertices are adjacent in $\Gamma$ if and only if they are adjacent in $\Gamma^{\prime}$. As Aut $\Gamma$ is primitive on $V \Gamma$ and Aut $\Gamma \leq$ Aut $\Gamma^{\prime}$, it follows that Aut $\Gamma^{\prime}$ must be primitive on $V \Gamma$, and hence $\Gamma^{\prime}$ is a primitive graph. Since $\Gamma$ has connectivity one, the same is true of $\Gamma^{\prime}$, so we may apply Theorem 3.2 to deduce the lobes of $\Gamma^{\prime}$ are primitive, pairwise isomorphic and each has at least three vertices. Now, given a lobe $\Lambda$ of $\Gamma$, there is a lobe $\Lambda^{\prime}$ of $\Gamma^{\prime}$ such that $V \Lambda=V \Lambda^{\prime}$. Therefore, the lobes of $\Gamma$ have at least three vertices, and are primitive but not automorphism-regular by Theorem 2.6.

It remains to show they are pairwise isomorphic. Fix some lobe $\Lambda$ of $\Gamma$ and an $\operatorname{arc}(\alpha, \beta) \in A \Lambda$. Let $\Gamma_{1}$ be the digraph $\left(V \Gamma,(\alpha, \beta)^{\text {Aut } \Gamma}\right)$. As Aut $\Gamma$ is primitive, this digraph is a connected subgraph of $\Gamma$. Thus, every lobe of $\Gamma$ must contain an arc in $A \Gamma_{1}$. Furthermore, if $\Delta$ is a lobe of $\Gamma$, then any automorphism of $\Gamma$ mapping the arc $(\alpha, \beta)$ to an arc in $\Delta$ must map $\Lambda$ to $\Delta$. Since $\Gamma_{1}$ is arc-transitive, the lobes of $\Gamma$ must be pairwise isomorphic.

It is now a simple exercise to classify those vertex-transitive digraphs with connectivity one which are counterexamples to the application of Jung and Watkins' result to digraphs without modification. Any such counterexample must be one of two types: digraphs that satisfy the conditions of Jung and Watkins' theorem, but are nevertheless imprimitive; and digraphs that are primitive, but fail to satisfy Jung and Watkins' characterisation.

Since the conditions given in Theorem 3.3 under which one may be certain a vertex-transitive digraph with connectivity one is primitive include the corresponding conditions given in Jung and Watkins' theorem, no primitive digraph with connectivity one is of the latter type. Thus, any counterexample must be imprimitive, yet still satisfy the conditions of Jung and Watkins result. We begin with a lemma.

Lemma 3.4 For each prime $p$, a digraph $\Lambda$ on $p$ vertices is a directed cycle if and only if its automorphism group is the cyclic group $C_{p}$ of order $p$.

Proof Suppose $p$ is prime and $\Lambda$ is a digraph on $p$ vertices. If $\Lambda$ is a directed cycle then clearly its automorphism group is $C_{p}$.

Conversely, suppose $\Lambda$ has automorphism group $C_{p}$. Without loss of generality, we may label the vertices of $\Lambda$ as integers $0,1, \ldots, p-1$ such that $(0,1)$ is an arc in $\Lambda$ and $C_{p}$ is generated by the permutation $\rho$ with

$$
i^{\rho}=i+1 \bmod p .
$$


Since $C_{p}$ is transitive on $V \Lambda$, for some set $J \subseteq\{1, \ldots, p-1\}$ we may write

$$
A \Lambda=\bigcup_{j \in J}(0, j)^{C_{p}} .
$$

Note that $1 \in J$. Let $\sigma$ be a permutation of $V \Lambda$ fixing the vertex 0 and fixing $J$ setwise. Choose $(a, b) \in A \Lambda$ and observe $(a, b)=(0, j)^{\rho^{k}}$ for some $j \in J$ and some integer $k$. Now $(a, b)^{\rho^{-k} \sigma}=(0, j)^{\sigma}=\left(0, j^{\prime}\right)$ for some $j^{\prime} \in J$, and $\left(0, j^{\prime}\right) \in A \Lambda$. Thus $\sigma$ lies in Aut $\Lambda$ and, if $J$ contains at least two elements, is not in $C_{p}$. Whence, $J=\{1\}$ and $\Lambda$ is a directed cycle.

Recall that any counterexample to the unmodified extension of Jung and Watkins' result to digraphs must be imprimitive, yet still satisfy the conditions of their theorem. By Theorem 3.3, all such digraphs must have automorphism-regular primitive lobes which are pairwise isomorphic, with each possessing at least three vertices. Let $\Gamma$ be such a digraph.

If $\Lambda$ is a lobe of $\Gamma$, then Theorem 1.1 tells us that Aut $\Lambda$ is cyclic of prime order $p$. Since this group is transitive, it implies that $\Lambda$ must have precisely $p$ vertices. Thus $\Lambda$ is a $p$-vertex digraph whose automorphism group is the cyclic group $C_{p}$ of order $p$, and is necessarily a directed cycle by Lemma 3.4.

Conversely, we note that for any odd prime $p$, a vertex-transitive digraph with connectivity one whose lobes have $p$ vertices and automorphism group $C_{p}$, satisfies the primitivity conditions given by Jung and Watkins for graphs.

Thus the counterexamples to the unmodified extension of Jung and Watkins' result are precisely those digraphs whose lobes have an odd prime $p$ number of vertices, and are directed cycles. This is summarised in our concluding theorem. Here the undirected graph associated with the digraph $\Gamma$ is the graph with vertex set $V \Gamma$ and edge set $\{\{\alpha, \beta\} \mid(\alpha, \beta) \in A \Gamma$ or $(\beta, \alpha) \in \mathrm{A} \Gamma\}$.

Theorem 3.5 If $\Gamma$ is a vertex-transitive imprimitive digraph with connectivity one, then its associated (undirected) graph is primitive if and only if the lobes of $\Gamma$ are pairwise isomorphic directed p-cycles, for some odd prime $p$.

Acknowledgements This paper contains parts taken from the author's DPhil thesis, completed under the supervision of Peter Neumann at the University of Oxford. The author would like to thank Dr Neumann for his tireless enthusiasm and helpful suggestions. The author would also like to thank the EPSRC for generously funding this research, and the two referees who strengthened this paper with their advice.

\section{References}

1. Bhattacharjee, M., Macpherson, D., Möller, R.G., Neumann, P.M.: Notes on Infinite Permutation Groups. Lecture Notes in Mathematics, vol. 1698. Springer, Berlin (1998)

2. Imrich, W.: Graphen mit transitiver Automorphismengruppe. Monatsh. Math. 73, 341-347 (1969)

3. Jung, H.A., Watkins, M.E.: On the structure of infinite vertex-transitive graphs. Discrete Math. 18, 45-53 (1977) 